\title{
A new building mask using the gradient of heights for automatic building extraction
}

\author{
Fasahat Ullah Siddiqui \\ Faculty of Information Technology \\ Monash University \\ Clayton Vic 3800 Australia \\ fasahat.siddiqui@monash.edu \\ Phone: +61351228020
}

\author{
Mohammad Awrangjeb \\ School of Info. \& Comm. Tech. \\ Griffith University \\ Nathan QLD 4111 Australia \\ m.awrangjeb@griffith.edu.au \\ www.ict.griffith.edu.au/awrangjeb/
}

\author{
Shyh Wei Teng \\ and Guojun Lu \\ Faculty of Science and Technology \\ Federation University Australia \\ Churchill Vic 3842 Australia \\ \{shyh.wei.teng, guojun.lu\}@federation.edu.au
}

\begin{abstract}
A number of building detection methods have been proposed in the literature. However, they are not effective in detecting small buildings (typically, $50 \mathrm{~m}^{2}$ ) and buildings with transparent roof due to the way area thresholds and ground points are used. This paper proposes a new building mask to overcome these limitations and enables detection of buildings not only with transparent roof materials but also which are small in size. The proposed building detection method transforms the non-ground height information into an intensity image and then analyses the gradient information in the image. It uses a small area threshold of $1 \mathrm{~m}^{2}$ and, thereby, is able to detect small buildings such as garden sheds. The use of non-ground points allows analyses of the gradient on all types of roof materials and, thus, the method is also able to detect buildings with transparent roofs. Our experimental results show that the proposed method can successfully extract buildings even when their roofs are small and/or transparent, thereby, achieving relatively higher average completeness and quality.
\end{abstract}

\section{INTRODUCTION}

Many researchers have been working in automatic building detection recently to meet a number of interesting applications such as disaster monitoring, real estate, national security and public service [1], [2], [3]. However, the automatic extraction of buildings from remotely sensed data is still challenging due to complexities like building structure variability, scene complexity (highly-dense vegetation, occluded buildings and hilly terrain), poor acquisition of data, and mismatching of data resolution from different sources.

In general, two different sources of data, i.e., Light Detection And Ranging (LiDAR) and photogrammetric imagery, are mainly used for building extraction. The main benefit of using a single source data, i.e., LiDAR data or imagery alone, is that the data mismatching problem is avoided. The use of high resolution (typically, 5 to $10 \mathrm{~cm}$ ground sampling distance) aerial imagery alone offers a great source of rich information with the increase of scene complexity. Shadows and occlusions are always among the main problems with using the imagery alone. Moreover, the colour and amount of vegetation change with time, especially trees may change colour and/or loose leaves in winter. In contrast, since LiDAR is an active sensing system, shadowing and darkness (say, due to cloudy weather or night acquisition of data) are not at all problems with using the point cloud data alone. The recent LiDAR systems are able to capture highly dense point clouds, typically more than 50 points $/ \mathrm{m}^{2}$, and therefore provide rich information. In addition, occlusions due to neighbouring tall objects can be easily circumvented by combining multi-pass point clouds, which are captured at different angles and/or flight lines. In fact, while building extraction from high resolution aerial imagery alone is semiautomatic, building extraction using LiDAR data alone can be made fully automatic [3].

Generally, the LiDAR-based building extraction methods segment the LiDAR point cloud data into building and nonbuilding groups using 2D or 3D features such as slope, direction, and neighbourhood connectivity. One approach is to segment the LiDAR points applying a rule-based method. For example, Sampath and Shan [4] used the Eigen analysis of each LiDAR point to determine its planarity. Only the planar points are further segmented using a customised fuzzy k-means algorithm. This clustering algorithm is unstable and produces different results for different initial seed points. In case of improper selection of initial seed points, the solution may converge to local minima [5]. In addition, the proposed clustering algorithm was verified with only one example that contains a large building. Thus, it is unknown whether this method works on small buildings.

Awrangjeb and Fraser [6] initially clusters the LiDAR data into two groups based on a height threshold. The LiDAR points which are above the threshold are marked as nonground points. These points are further divided based on a neighbourhood feature, i.e. co-planarity of points. Using this co-planarity information, the planar roof segments are extracted [7]. Finally, a rule-based procedure is followed to filter out the planes representing trees. Experimental results showed that this method failed to extract small and transparent buildings [6], [7].

Abdullah et al. [7] used the Delaunay triangulation to determine the neighbouring points of non-ground points. This neighbourhood information is employed in the Eigen analysis to measure the planarity of the non-ground points. Later, a region growing method is applied on the non-ground coplanar LiDAR points to extract $3 \mathrm{D}$ building planes. Initially, the seed points of the region growing method are defined by taking the mid-points of a LiDAR point cluster at different height levels. The grown regions at different height levels are merged if they satisfy a single plane equation. Finally, all the extracted planes are combined into bigger regions 
which represent potential distinct buildings. A rule-based post processing procedure based on used point ratio, object shape information and height gap is finally applied to remove trees. However, the performance of this method is sensitive to the initial seed points [8]. In addition, shape analysis and used point ratio in the post-processing procedure remove small and transparent buildings, respectively.

In Awrangjeb and Fraser [9], the plane fitting analysis is used on the non-ground LiDAR points to determine the 3D plane of buildings for building extraction. Initially, all the possible building edges are extracted from the colour image, Normalized Difference Vegetation Index (NDVI) image, texture image and the non-ground mask. Later, these edges are classified into building edges and ridges. The regions on the building side of building edges are marked and are extended until no new non-ground LiDAR points fitting the same plane equation are found. However, this method needs many preset parameter values to work effectively, which is difficult to set and subjective. Shape analysis is used as a post processing step to remove the small buildings. In addition, the transparent buildings are not extracted while generating the primary building mask and and therefore these buildings are missed in the final extraction [7].

The methods in [6] and [9] use the ground points to produce a primary building mask (i.e. initial building cue). Therefore transparent buildings which have large number of ground points are removed from the primary building mask and are excluded from further processing. To our knowledge, Region Growing based Building Extraction (RGBE) method by Abdullah et al.[7] is a promising rule-based method that is not using the primary building mask and processes transparent building points in building planes extraction step.

In photogrammetric and remote sensing research, knowing the prominent (or principal) directions of buildings is a prerequisite in many early studies, particularly for building detection [10] and building outline regularisation [11]. Awrangjeb et al. [10] assume that buildings in a given local area are oriented in one prominent direction. Nonetheless, such an area may not be uniquely defined. Moreover, in practice there may be more than one principal directions for a complex building shape. Awrangjeb and Fraser [11] have later used the long image lines (at least $6 \mathrm{~m}$ ) in order to obtain multiple prominent directions of a building. In this paper, a new algorithm for determination of prominent directions of buildings based on extracted images lines has been proposed.

In order to automatically extract buildings which are transparent and of greater range of sizes (including the smaller buildings), a new Gradient-based Building Extraction (GBE) method is proposed in this paper. The proposed GBE method proposes a new building mask based on the non-ground point cloud data. It first obtains the non-ground LiDAR points on buildings and trees by applying a height threshold to the input point cloud data. The non-ground points are then used to generate height intensity images at different prominent directions of buildings. Prominent directions are determined by analysing straight lines extracted from the input high resolution aerial imagery. A grid is set at each prominent direction and the non-ground points are sampled based on the mean height of the points within each cell in the grid. The mean calculation reduces the height error in the LiDAR data which also helps in extraction of the transparent buildings. The gradient of heights is then calculated in two directions i.e. $X$ and $Y$ axes of the grid. The pixels whose gradient values are constant in at least one direction are marked as pixels of building planes or regions. In contrast, the pixels whose gradient values are not constant in both directions are marked as pixels of trees. The gradient of heights estimation thus removes a major part of vegetation that shows high height variations. As mentioned above, shape analysis causes the removal of small buildings, therefore, post processing steps which include variance and texture analysis are applied to remove more vegetation while preserving small buildings. Since the proposed GBE method generates the building mask using the non-ground points, the transparent buildings are successfully extracted.

The remainder of this paper is organized as follows: Section II outlines the proposed GBE method. Experimental setup and parameters settings are described in Section III. Section IV presents the qualitative and quantitative results of the GBE method compared with the benchmark method [7]. Finally, Section $\mathrm{V}$ concludes the paper with future research direction.

\section{Proposed Method}

The proposed GBE method produces an intensity image using the height information from LiDAR data. The derived image is generated by overlaying a grid on non-ground LiDAR points in a prominent direction of buildings. Next, the mean height of the LiDAR points in each cell of the grid is assigned as the image pixel of that grid. The mean value reduces the height variation on a transparent building and this helps in extracting the transparent building pixels. The gradient is then calculated using the derived image in two defined directions in order to evaluate if there is a constant change of the pixel values. The constant change in pixel value determines the building regions (planes). As mentioned earlier, the shape analysis may delete small buildings. Therefore, the proposed GBE method uses texture analysis (i.e. entropy) and variance to remove the trees and to keep the small buildings. Their adoption is based on the observation that while the height value of points on trees has a nature of randomness, that on buildings shows a regular change because of flat and sloppy planes. Whereas, a high entropy and a high height variance indicate a tree region, a low entropy and a small height variance indicate a building region.

A flow diagram of the proposed GBE method is shown in Fig. 1. In this section, the four main steps are explained with the help of a sample data set, shown in Fig. 2. While Fig. 2(a) shows the LiDAR point cloud overlaid on the aerial imagery, Fig. 2(b) shows the non-ground points with height at least 1 $\mathrm{m}$ above the ground.

\section{A. Determination of prominent directions of buildings}

Straight lines are extracted from the input aerial imagery following the procedure introduced in [10], [12]. Canny edge algorithm is first applied to find irregular (zigzag) edges. Corners are then detected on the extracted edges. A straight line is finally fitted between the two consecutive corners on each extracted edge. Fig. 2(c) shows all the extracted lines from the sample scene. As can be seen, not all the extracted image lines are building lines, i.e., building edges and ridges. 


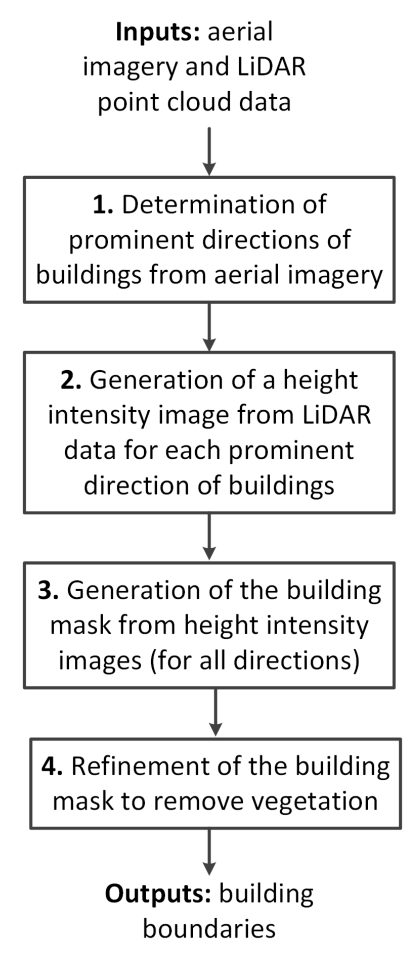

Fig. 1: Four main steps of the proposed GBE method.

In order to determine the prominent directions of buildings, irrelevant image lines have to be removed.

Many researchers assume buildings are at least $3 \mathrm{~m}$ long [6][7][9], therefore, we choose a $3 \mathrm{~m}$ threshold to remove all short lines $\left(d_{\max }=3 \mathrm{~m}\right)$. Then, lines on the ground are removed if there is no non-ground points (see Fig. 2(b)) on both sides of an extracted line. Moreover, lines on and around a vegetation can be removed by analysing NDVI and texture information [12]. If there is a high NDVI and texture information on at least one side of an extracted line, then this line is eliminated. Fig. 2(d) shows the surviving image lines at this moment. The two (ground) lines near the right-bottom corner survive due to non-ground LiDAR points reflected from the neighbouring cloth hoist and vegetation (see Fig. 2(b)).

Now, in order to know the prominent directions, a histogram analysis based on angles of image lines is carried out. The angle is measured in $2 \mathrm{D}$ space with respect to the positive $x$-axis, i.e., horizontal line passing through the top-left corner of the image. The histogram has a range of angles from $-180^{\circ}$ to $180^{\circ}$. It consists of 64 bins, i.e., the distance between the successive bins is $5.625^{\circ}$ ( $\frac{\pi}{32}$ radian). The small bin distance in fact allows buildings to have close principal directions, even within a small area. Once the lines are put into the bins based on their angles, the mean angle of lines in a bin is considered as a candidate for prominent angles of buildings. There are such eight groups of lines as shown by different colours in Fig. 2(e).

As can be seen within orange coloured ellipses in Fig. 2(e), some of the bins are close to each other due to small bin distance. If some lines in one bin are physically close (within $2 d_{\max }$ ) to the lines of a neighbouring bin, they are considered to be in the same bin (removed from the minority bin and put into the majority bin). Moreover, some bins are perpendicular to others. In that case, the bin that has less number of lines is not considered anymore, as its direction can be easily estimated from the direction of the bin that has more number of lines. For example, in Fig. 2(e) the green bin (6 lines) is removed in favour of the red bin (8 lines). Consequently, there are only 3 bins left as shown in Fig. 2(f) in three different colours (red, green and blue). The means of angles in these bins are considered to be the final prominent directions of buildings and ranked in order of the bin size, i.e., the number of lines. The three estimated prominent angles are $-6.4^{\circ}$ (red bin with 8 lines), $-55.7^{\circ}$ (green bins with 3 lines) and $-83.9^{\circ}$ (blue bin with 1 lines).

The first prominent direction is a true direction of the building in the sample scene. The second prominent direction indicates the ridge angles of the same building. Thus, it is a false prominent direction. The third prominent direction is also false and obtained due to non-ground points (on vegetation) on a side of the blue line shown in Fig. 2(f). Since, the false prominent directions do not negatively impact the proposed building detection method, they are not removed.

\section{B. Generation of a height intensity image}

A height intensity image is created for each estimated prominent direction of buildings using the height from the nonground point cloud data. A grid with a resolution of twice the maximum LiDAR point-spacing is aligned with a prominent direction of buildings and overlaid on the non-ground LiDAR data (see Fig. 3(a)). If there is no points in a cell (i.e., ground), the cell is marked and will not be considered for gradient estimation discussed below. Otherwise, the cell is assigned the mean height of the points within the cell. The advantage of using the mean is to reduce the height error in LiDAR data so that the transparent buildings can be extracted.

The idea here is - since planes on buildings may be oriented in different directions, the rotation of the grid to a true prominent direction will align the rows and columns (cells) of the grid towards the direction of the slope of the corresponding roof planes. Unlike the flat plane, a sloppy plane will align (near) constant height values along the rows (or columns) but (near) a constant change of heights along the columns (or rows). In this case, the change of heights depends on the slope of the plane and the point cloud density. For the flat plane, irrespective of the grid orientation, the generated intensity image should remain (almost) the same, i.e., (near) constant height values along the rows and columns. However, the rotation of the grid to a false prominent direction will not align the rows and columns (cells) of the grid properly towards the direction of the slope of any sloppy roof planes. Thus, there will be random change of heights in the intensity image.

As illustrated in Fig. 3(a), the grid has been oriented towards the (true) prominent direction of $-6.4^{\circ}$. It can be observed that all corresponding roof planes are now properly aligned to the grid. In contrast, Fig. 3(c) shows the grid orientation for the (false) prominent direction of $-83.9^{\circ}$ and we see that none of the roof planes are properly aligned with the grid. 

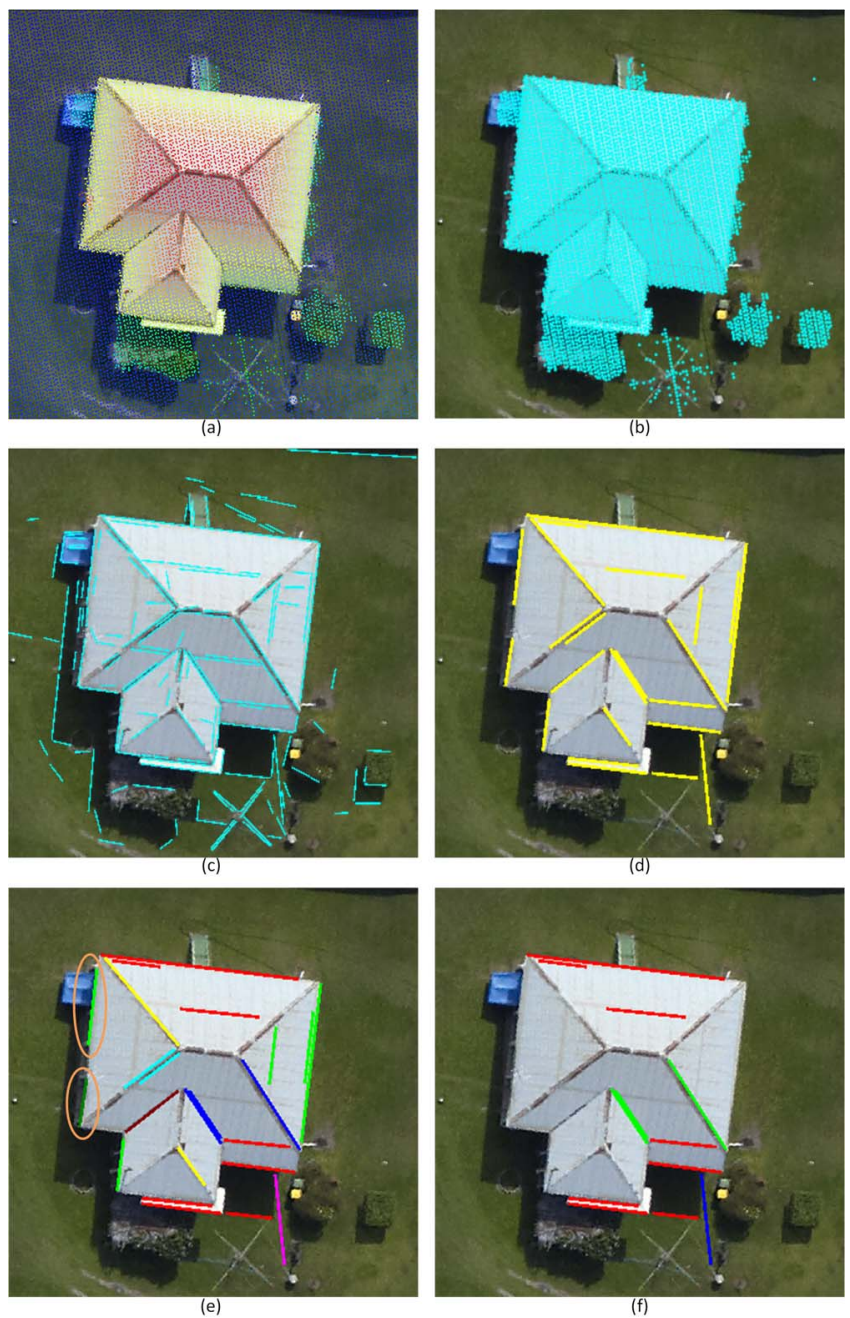

Fig. 2: Extraction and classification of image lines for a sample data set: (a) inputs - point cloud data overlaid on an aerial image (points are shown in colours where red colour indicates points with high height values and blue colour indicates points with low height values), (b) non-ground points, (c) extracted lines from the input image, (d) non-ground long lines, (e) lines into eight histogram bins (shown in different colours), and (f) lines at three prominent directions.

It should be noted that for vegetation, on which usually random height values are expected, for both the true and false prominent direction of buildings, there will also be random change of heights in the intensity image.

\section{Generation of the building mask}

The gradient is calculated (in both $X$ and $Y$ directions) for each intensity image using a differential function. The gradient here represents the slope and tangent of the intensity changes among the pixels in the intensity image. For a true prominent direction, the corresponding intensity image will result in a (near) constant rate of change in height in $X$ or $Y$ direction for the relevant roof planes, whereas for any trees it will result in a high and non-constant rate of change in height. A threshold of $0.2 \mathrm{~m}$ is applied to identify the roof planes. A region with a rate of change in height less than $0.2 \mathrm{~m}$ is detected and marked as buildings in the building mask. Fig. 3(c) shows the building mask for the true prominent direction of buildings, while Fig. 3(d) illustrates the building mask for a false prominent direction of buildings. As can be seen, the gradient calculation is vital to extract true buildings and remove vegetation in the proposed building extraction method.

\section{Refinement of the building mask}

As mentioned earlier, the refinement process is used for removal of vegetation that may still survive in the building mask. However, the small building regions may also be removed if a shape (i.e., using a large area threshold) analysis is included into the refinement process [6], [7]. Therefore, entropy (texture analysis) and variance parameters are used in the proposed GBE method to remove the trees. As it is mentioned in Section II, the entropy and variance are adopted based on the principle that trees are rich in texture and exhibit large height variance as compared to buildings. In order to calculate the entropy at a pixel $(i, j)$ of the input aerial image a window of $9 \times 9$ is used on the gray-scale version of the image using the function

$$
\text { Entropy }=-\sum \sum P(i, j) \log P(i, j) \text {, }
$$




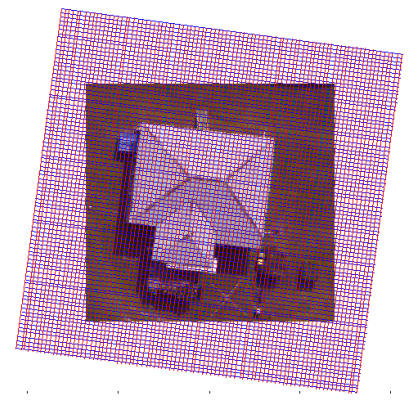

(a)

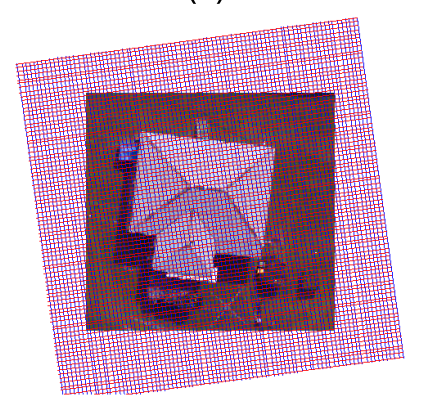

(c)

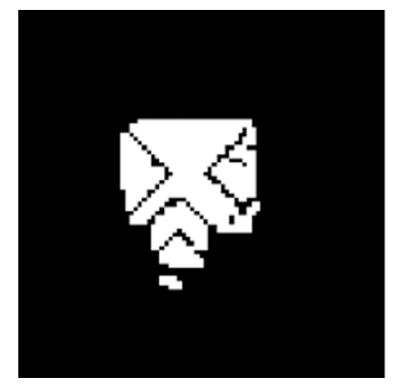

(e)

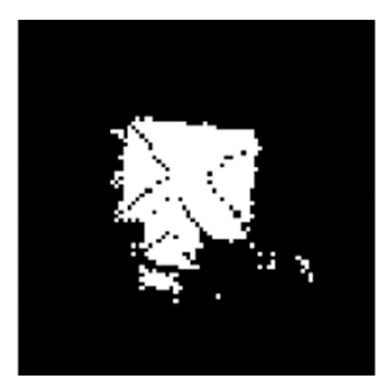

(b)

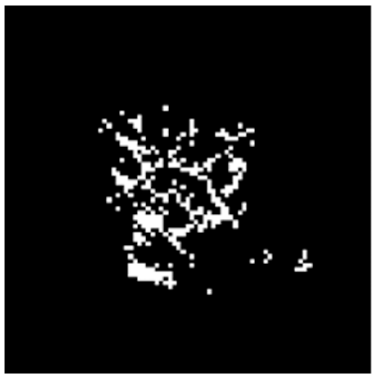

(d)

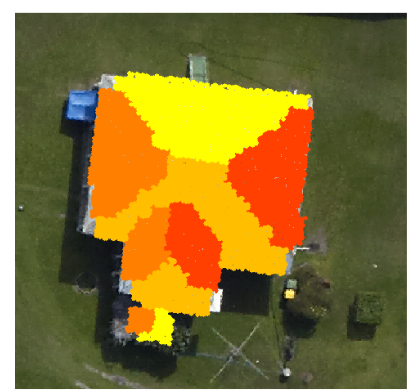

(f)

Fig. 3: (a) Grid produces in the true direction of buildings and (b) its gradient based building Mask; (c) Grid produces in a false direction of buildings and (d) its gradient based building Mask; (e) Building Mask after applying refinement process and (f) building roof planes.

where, $P$ is the pixel value. Similarly, the height variance is calculated from the non-ground point-cloud data. In this case, the window size is equal to twice the maximum LiDAR point spacing. Two thresholds, $0.5 \mathrm{~m}$ for height variance and 0.8 for entropy, are set to differentiate building from tree. Pixels in the mask where trees are indicated in any building masks are made black.

The building masks may still contain some noisy pixels sparsely located here and there. These noisy planes are eliminated by applying the Morphological filter with $1 \mathrm{~m}$ structuring element. Finally, the building mask, shown in Fig. $3(\mathrm{e})$, is produced by combining the building masks (by simple OR operation). Fig. 3(f) shows individual roof planes for the sample test scene.

\section{EXPERIMENTAL SETUP}

\section{A. Benchmark data set}

Two areas Aitkenvale (AV) and Harvey Bay (HB) of two Australian sites have been used to evaluate the performance of the proposed GBE method. AV has a point density of 29 points $/ \mathrm{m}^{2}$ and it covers an area of $214 \mathrm{~m} \times 159 \mathrm{~m}$, while HB has a point density of 12 points $/ \mathrm{m}^{2}$ and it covers around 108 $\mathrm{m} \times 104 \mathrm{~m}$ in area.

\section{B. Evaluation system}

To evaluate the performance of our proposed GBE method, the reference benchmark is obtained using the Barista software [13]. In addition, a threshold free evaluation system is employed [14]. In this evaluation system, three categories of evaluation metrics, i.e., object-based, pixel-based and geometricalbased, are used. A number of metrics are used in each category. The object-based metrics, i.e., completeness $\left(C_{m}\right)$, correctness $\left(C_{r}\right)$, and quality $(Q)$, estimate the performance by counting the number of buildings, whereas the pixel-based metrics, i.e., completeness $\left(C_{m p}\right)$, correctness $\left(C_{r p}\right)$ and quality $\left(Q_{p}\right)$, estimate the performance by counting the number of pixels of the extracted objects. In addition, the geometric metric, i.e., Root Mean Square Error ( $R M S E)$, indicates the accuracy of 


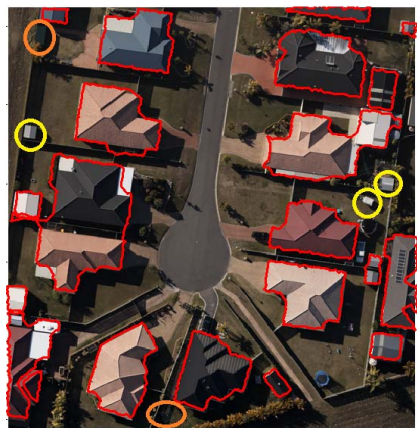

(a)

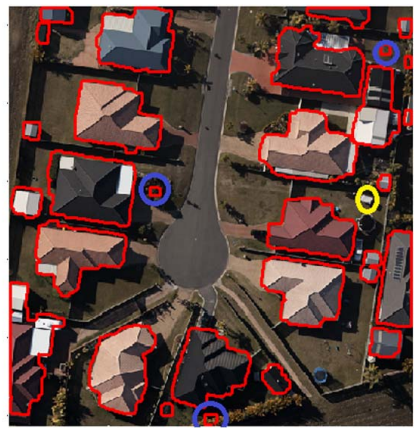

(b)

Fig. 4: Qualitative results after applying the (a) RGBE and (b) GBE on HB data.

TABLE I: Parameters used in the proposed GBE method.

\begin{tabular}{l|l|l}
\hline \hline Parameters & Values & Sources \\
\hline \hline Height threshold & $1 \mathrm{~m}$ & {$[7]$ and [3] } \\
\hline Minimum straight line length $d_{\max }$ & $3 \mathrm{~m}$ & {$[10],[12]$ and [3] } \\
\hline Bin distance & $5.625^{\circ}$ & {$[3]$} \\
\hline Grid cell size & $2 d_{\max }$ & {$[3]$} \\
\hline Structuring element size & $1 \mathrm{~m}$ & {$[3]$ and [7] } \\
\hline Height tolerance & $0.2 \mathrm{~m}$ & this paper \\
\hline Variance & $0.5 \mathrm{~m}$ & {$[15]$} \\
\hline Entropy threshold & 0.8 & {$[12]$} \\
\hline \hline
\end{tabular}

the extracted building boundaries with respect to the reference building boundaries. The complete description of the above mentioned metrics are defined in [14].

\section{Parameter settings}

Table I shows the list of parameters used by the proposed GBE method. Most of these parameters are adopted from the existing methods for building extraction. For example, height and the minimum straight line length thresholds are commonly used parameters in the literature for building cue extraction [7], [3], [10], [12]. Similarly, the other two parameters, i.e., grid/window length and degree range, have been used in literature for extracting the building plane [3]. While structuring element size of the morphological opening filter, variance and texture threshold have been used in literature for removing the noise and vegetation [3], [10], [12], [15]. In this paper, height tolerance threshold is the only new defined parameter. The height tolerance threshold is set to $0.2 \mathrm{~m}$.

\section{EXPERIMENTAL RESULTS}

Two test areas are shown in Figs. 4 and 5 with the extracted building boundaries. Qualitative and quantitative analyses are performed to evaluate the performance of the proposed GBE method. We have also compared the performance of the proposed GBE method with Region Growing based Building Extraction (RGBE) method [7], as this method is one of the most promising rule-based building extraction methods currently in the literature. This method does not use the primary building mask and process the transparent building points in building plane extraction step. Evaluating qualitatively, all transparent buildings and more small buildings are successfully extracted by the GBE method as compared to the RGBE
TABLE II: Evaluation for the Aitkenvale (AV) and Harvey Bay (HB) in percentage

\begin{tabular}{|c|c|c|c|c|c|c|c|}
\hline \multirow[t]{2}{*}{ Indices } & \multirow[t]{2}{*}{ Method } & \multicolumn{3}{|c|}{$\begin{array}{l}\text { Pixel based } \\
\text { Evaluation }\end{array}$} & \multicolumn{3}{|c|}{$\begin{array}{c}\text { Object based } \\
\text { Evaluation }\end{array}$} \\
\hline & & HB & $\mathbf{A V}$ & Avg & AV & HB & Avg \\
\hline \multirow[b]{2}{*}{$C_{m}$} & baseline & $\overline{991.5}$ & 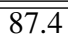 & 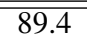 & $\overline{882.0}$ & 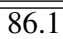 & 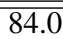 \\
\hline & $\mathrm{GBE}$ & 96.1 & 83.3 & 89.7 & 100 & 84.6 & 92.3 \\
\hline \multirow[b]{2}{*}{$C_{r}$} & baseline & 92.4 & 84.5 & 88.4 & 100 & 87.0 & 93.5 \\
\hline & GBE & 86.5 & 94.8 & 90.6 & 85.7 & 84.2 & 84.9 \\
\hline \multirow[b]{2}{*}{$Q$} & baseline & 85.2 & 75.4 & 80.3 & 84.0 & 73.0 & 78.5 \\
\hline & GBE & 83.6 & 79.7 & 81.6 & 83.0 & 74.0 & 78.5 \\
\hline \multirow[b]{2}{*}{$R M S E$} & baseline & 1.08 & 2.43 & 1.755 & - & - & - \\
\hline & GBE & 0.91 & 1.26 & 1.085 & - & - & - \\
\hline
\end{tabular}

method. The transparent buildings and small buildings, which are not extracted by each method, are highlighted with orange and yellow circles, respectively, in Figs. 4 and 5.

In addition, evaluating quantitatively, $C_{m}, C_{r}$ and $Q$ values in Table II also show that the proposed GBE method is more robust in extracting buildings of bigger range of sizes in both $\mathrm{HB}$ and $\mathrm{AV}$ areas. In addition, the GBE method is also robust in extraction of more transparent buildings. $C_{m}$ values clearly show that a greater total area of buildings is extracted by the GBE method. However, the object based correctness for GBE is comparatively low. This is due to the extraction of small false buildings, which are highlighted with blue circles in Figs. 4 and 5. In addition, the average completeness of the GBE method is also higher than that of the RGBE method by $0.3 \%$ in pixel based and $8.25 \%$ in object based evaluations. The average quality of the GBE is also improved by $1.35 \%$ in pixel based evaluation. Furthermore, the GBE method shows lower $R M S E$ as compared to the RGBE method, which indicates the robustness of GBE in terms its accuracy in extracting building geometry.

\section{CONClusion}

In this paper, a new building extraction method has been proposed. This gradient-based method is more robust in extracting building of bigger range of size as well as transparent buildings. The proposed method is compared to a state-ofthe-art method for evaluation. The experimental results show that the proposed method is more effective in extracting 


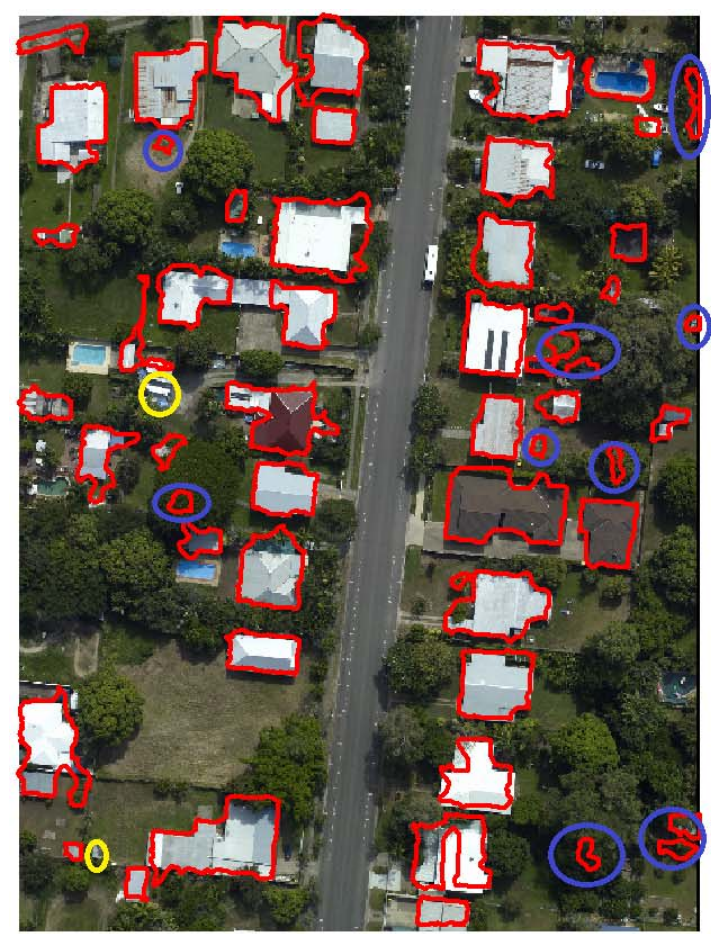

(a)

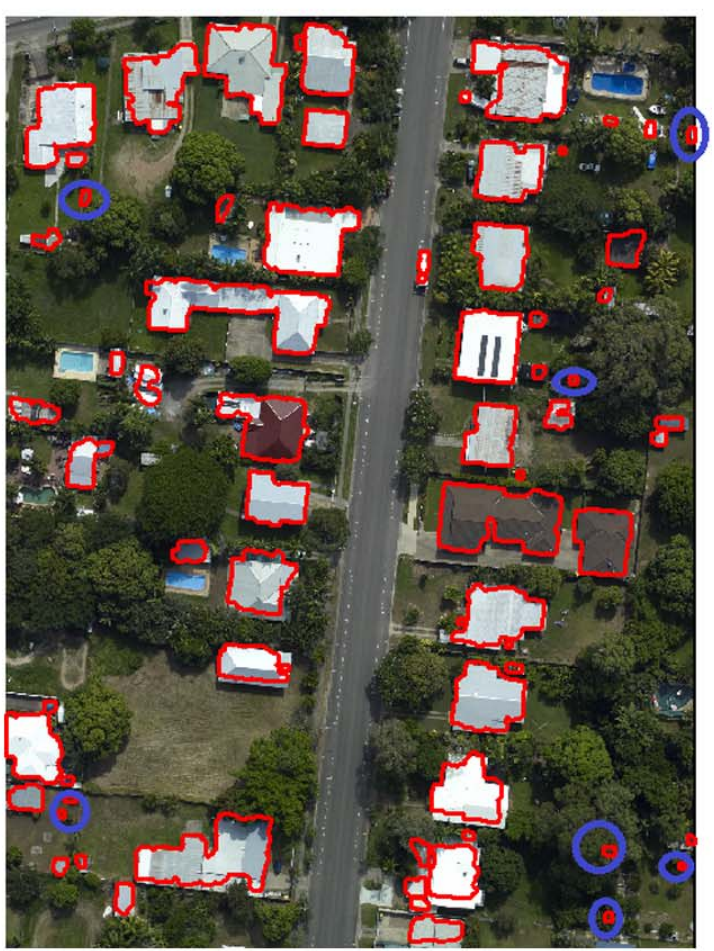

(b)

Fig. 5: Qualitative results after applying the (a) RGBE and (b) GBE on AV data.

all types of buildings, i.e. small and transparent buildings. Comparing the quantitative results of the two methods, the GBE method's average completeness is $0.3 \%$ higher in pixelbased and $8.25 \%$ higher in object-based evaluation. Its average quality is also $1.35 \%$ higher in pixel-based evaluation. The future work includes 3D reconstruction of building models from high density point cloud data.

\section{ACKNOWLEDGMENT}

The authors would like to thank Ergon Energy (www.ergon.com.au) for providing the Australian data sets. Dr. Awrangjeb is a recipient of the Discovery Early Career Researcher Award by the Australian Research Council (project number DE120101778).

\section{REFERENCES}

[1] G. Sohn and I. Dowman, "Data fusion of high-resolution satellite imagery and lidar data for automatic building extraction," ISPRS Journal of Photogrammetry and Remote Sensing, vol. 62, no. 1, pp. 43-63, 2007.

[2] Y. Li, H. Wu, R. An, H. Xu, Q. He, and J. Xu, "An improved building boundary extraction algorithm based on fusion of optical imagery and lidar data," Optik-International Journal for Light and Electron Optics, vol. 124, no. 22, pp. 5357-5362, 2013.

[3] M. Awrangjeb and C. S. Fraser, "Automatic segmentation of raw lidar data for extraction of building roofs," Remote Sensing, vol. 6, no. 5, pp. 3716-3751, 2014.

[4] A. Sampath and J. Shan, "Segmentation and reconstruction of polyhedral building roofs from aerial lidar point clouds," IEEE Transactions on Geoscience and Remote Sensing, vol. 48, no. 3, pp. 1554-1567, 2010.

[5] F. U. Siddiqui, N. A. M. Isa, and A. Yahya, "Outlier rejection fuzzy c-means (orfcm) algorithm for image segmentation," Turkish Journal of Electrical Engineering \& Computer Sciences, vol. 21, no. 6, pp. 1801-1819, 2013.

[6] M. Awrangjeb and C. S. Fraser, "Rule-based segmentation of lidar point cloud for automatic extraction of building roof planes," ISPRS Ann. Photogramm. Remote Sens. Spat. Inf. Sci, vol. 2, no. 3/W1, pp. 1-6, 2013.

[7] S. Abdullah, M. Awrangjeb, and G. Lu, "Lidar segmentation using suitable seed points for $3 \mathrm{~d}$ building extraction." ISPRS-International Archives of the Photogrammetry, Remote Sensing and Spatial Information Sciences, vol. 1, pp. 1-8, 2014.

[8] I. Avazpour, M. I. Saripan, A. J. Nordin, and R. S. A. R. Abdullah, "Segmentation of extrapulmonary tuberculosis infection using modified automatic seeded region growing," Biological procedures online, vol. 11, no. 1, pp. 241-252, 2009.

[9] M. Awrangjeb, C. Zhang, and C. S. Fraser, "Automatic extraction of building roofs using lidar data and multispectral imagery," ISPRS Journal of Photogrammetry and Remote Sensing, vol. 83, pp. 1-18, 2013.

[10] M. Awrangjeb, M. Ravanbakhsh, and C. S. Fraser, "Automatic detection of residential buildings using lidar data and multispectral imagery," ISPRS Journal of Photogrammetry and Remote Sensing, vol. 65, no. 5, pp. 457-467, 2010.

[11] M. Awrangjeb, "Using point cloud data to identify, trace and regularize the outlines of buildings," International Journal of Remote Sensing, vol. 37, 2016.

[12] M. Awrangjeb, C. Zhang, and C. S. Fraser, "Building detection in complex scenes thorough effective separation of buildings from trees," Photogrammetric Engineering \& Remote Sensing, vol. 78, no. 7, pp. 729-745, 2012.

[13] Barista. (2011) The barista software. [Online]. Available: www.baristasoftware.com.au

[14] M. Awrangjeb and C. S. Fraser, "An automatic and threshold-free performance evaluation system for building extraction techniques from airborne lidar data," Selected Topics in Applied Earth Observations and Remote Sensing, IEEE Journal of, vol. 7, no. 10, pp. 4184-4198, 2014.

[15] F. U. Siddiqui, S. W. Teng, G. Lu, and M. Awrangjeb, "An improved building detection in complex sites using the lidar height variation and point density," in Image and Vision Computing New Zealand (IVCNZ), 2013 28th International Conference of. IEEE, 2013, pp. 471-476. 